Transactions of the SDPS:

Journal of Integrated Design and Process Science

16 (2), 2012, 1-3

DOI 10.3233/jid-2012-0014

http://www.sdpsnet.org

\title{
Editorial: Tackling the Challenges of IT Services Design
}

Service design is the activity of planning and organizing people, infrastructure, communication and material components of a service in order to improve its quality and the interaction between service provider and customers (Mager, 2008). In services an activity usually involves people. It can be, for example, a class meeting that has to be attended by certain students, a flight that transports passengers, and an operation that has to be done by a surgeon on a given day. A service significantly involves customer inputs (Sampson \& Froehle, 2006). In other words, in order for a service to be produced, a customer has to present personally or he/she has to present his/her belongings or information.

Customer inputs are the root cause of the unique issues and challenges of service design. The literature has typically identified three general types of customer inputs (Wemmerlov, 1990): the customer's self, his belongings or other tangible objects and information. Customer-self inputs are common in services involving co-production (i.e., the employment of customer labor in the process) and in services involving the physical presence of the customer. Tangible belongings and physical objects are another type of input a customer can provide to the service process. For example, one's car is an essential input into the automobile repair service process. Customer-provided information is a third type of input to the service process. For example, a computing service process requires that customers provide their data as information input. Without that input the service production process cannot begin.

For IT Services, the customers' inputs are their information which can be their data to be processed or the transaction information regarding their business processes. To ensure quality, customer information has to be handled appropriately in the design of service processes. A service system should be responsive to changes from customer and accommodate them in a way that maintains service quality. The requirement of customer information in IT Services leads to dynamic service processes. The needed information is scattered among possibly a large number of customers. Collecting the information and keeping it up to date can be challenging tasks. Customers may ask to include additional tasks that are not anticipated, or to adapt to changes to several tasks, or to neglect certain tasks. The resources available for performing tasks are subject to changes as well. Certain resources can become unavailable, and additional resources may need to be introduced. In addition to accommodating customer changes, the presence of customer information also demands data security and confidentiality measures in IT Services design. This is specially the case when customers need to submit their private and sensitive corporate data in order to get the services performed. IT Services, especially those offered through public networks, expose the system to more attacks. An IT Service system should prevent unauthorized access to customer data; ensure that one customer's data is fully segregated from another customer's data; restore the data if a disaster occurs. In addition, there is a location related concern. Service providers should commit to storing and processing data in specific jurisdictions required by customers, which may require the design of distributed service systems allocated in various locations around the world. Furthermore, IT Services 
offered through Internet should be able to scale quickly to accommodate rapidly growing customer demands. The diversity of customer requirements also demands flexibility in service resource configuration. Service Oriented Architecture (SOA) provides a framework for composing a needed service from a group of components, which provides the flexibility required in IT Services. Cloud computing is an ideal service delivery infrastructure for dealing with the scalability issue of IT Service system design. Given its elastic nature in resource management, IT Services delivered through cloud computing can automatically scale up and down in response to the service load. In addition, this scaling also conserves resources and reduces the impact of data centers on the environment.

The papers selected in this issue address several aspects of IT Services design. The first paper, titled "Predicting the Future of IT Services with TRIZ", predicts the future generations of IT Services from the innovation management perspective. Gibson and Kasravi analyze the trends of future IT Services through a structured innovation model: Theory of Inventive Problem Solving (TRIZ). By reviewing the historical trends of IT Services, they mapped the history of IT Services to the four states of Technology Evolution Vector prescribed in TRIZ. Based on their analysis, current IT Services have passed the Rigid, Modular, and Programmable states and entered into the Autonomous state which is characterized by transparent to the user, highly automated, low cost, self-managed, and not people-intensive service design. Highly automated and transparent IT Services are necessary to effectively accommodate customer and environment changes in service provision and attract a lager customer base.

In their paper titled "Agent-Based System Design for Service Process Scheduling: Challenges, Approaches and Opportunities”, Dargahi et al. provided a survey of agent-based system design for service scheduling. The main focus of the agent-based approach is to address the challenges of dynamic environments in service scheduling. In services, there are typically no inventoried products, which make the service provider's capacity more sensitive to dynamic changes. The authors identified three major challenges to the design of service scheduling systems, namely distributed and dynamic environment, complicated objectives, and customers' private information. The survey focuses on four service scheduling domains: meeting, healthcare, transportation, and computing services. In addition to distributed dynamic environments, the paper also analyzes how mechanism design based approach, such as auctions, are used to elicit customers' private information given a strategic scheduling setting. Based on their review, the authors conclude that despite of many domain-specific designs in agent-based service process scheduling, there is a lack of general problem formulations, classifications, solution frameworks, and test beds.

By proposing a Stream-based NOvel class Detection for MALware (SNODMAL), the third paper by Thuraisingham et al., titled "Design and Implementation of a Data Mining System for Malware Detection”, considers security issues in IT Services design. SNODMAL is a specially designed data mining system which detects malwares. The paper describes the design of SNODMAL which includes system architecture, approaches for behavioral and binary feature extraction and selection, and models for data stream classification and novel class detection. At the heart of SNODMAL is a classification model which is used to classify data. Based on the class label, appropriate measures will be taken. The classification model is incrementally updated with feature data obtained from new malware executables. When a new malware instance is chosen as a training example, it will be analyzed and its behavioral and binary patterns will be recorded. The recorded patterns will be used to compute feature vectors. These vectors will be used to update the existing classification model.

With the advances in wireless communication and mobile technology, mobile computing has emerged to be a very important market for IT Services. Mobile computing delivers services to wherever the customers are, which integrates customer information into the service process in a real time or near real time manner. Through close integration and highly responsive interaction with service providers, mobile computing enables the building of dynamic and personalized service systems. The third paper by Kim et al., titled “Agent-Based Mobile Data Caching Strategies Using Data Significance”, studies data caching strategies in mobile IT Services with the aim of speeding up access to frequently used data and providing 
some level of access under network disconnection by temporarily replicating a portion of data into the memory of a mobile client. The authors suggested an agent-based mobile data access model. Two functions are proposed to calculate data significance scores in order to choose suitable data items for cache replacement or cache coherence. These functions synthetically reflect access term, access frequency and tendency, update frequency and tendency, and data item size distribution. Cache replacement and coherence algorithms are also proposed to make data caching decisions based on the significance scores.

To support the integration of highly distributed and heterogeneous precision farming databases, Moon et al., in their paper titled "An Intelligent Decision Support System for Cropland Suitability Evaluation”, designed an IT Service system based on the Service Oriented Architecture (SOA). The SOA is implemented using Web services technology. The proposed system delivers a recommendation service to carry out cropland suitability evaluation. The recommendation service is supported by three components that perform the knowledge discovery, refinement of relevant data formats, and data-driven estimation of significances-by-attributes. The authors also proposed data extraction functions for implementing the knowledge discovery process, data refinement methods for transforming naïve candidate datasets into unified ones, and the data-driven attribute significance quantification algorithm for quantifying the influence of attributes on the decision tree.

Given the rapid development of cloud computing technologies in recent years, IT Services providers now have the option of moving their services into the cloud. The idea of using cloud as service delivery platform is attractive since cloud-based services bring in customers the benefits of elastic computing resources, on-demand services, and cost savings. The pay-per-use model enables companies to grow their business quickly to satisfy market demand without huge initial investment on the IT infrastructure. However, moving IT Services into the cloud does not solve some fundamental challenges of IT Services design, such as those studied in the selected paper. Approaches that tackle those fundamental challenges in the context of cloud-based delivery platform are much needed in future IT Services design.

\section{References}

Mager, B. (2008). Service design. Design dictionary: Perspectives on design terminology, 354-357, M. Erlhoff and T. Marshall (eds), Basel, Birkhäuser

Sampson, S. E. \& Froehle, C. M. (2006). Foundations and implications of a proposed unified services theory. Production and Operations Management, 329-343

Wemmerlov, U. (1990). A taxonomy for service processes and its implications for system design. International Journal of Service Industry Management 1(3), 13-27

Guest Editor: Chun Wang

Concordia Institute for Information Systems Engineering Faculty of Engineering and Computer Science

Concordia University chun.wang@concordia.ca 